\title{
Evaluation of Organogenesis \& Morphogenesis of Kidney in Human Fetuses at Different Weeks of Gestation
}

\author{
Journal Of Medical Science And Clinical Research \\ IGM Publication \\ An official Publication of IGM Publication
}

Authors

\section{Ramendra Raman ${ }^{1}$, Ganpat Choudhary ${ }^{2},{\text { Rakesh } \mathrm{Sah}^{3} \text {, Astha Shrestha }}^{4}$ Srijana Shrestha ${ }^{5}$}

${ }^{1,3}$ Lecturer, Department of Anatomy, Kantipur Dental college Teaching Hospital \& Research Center, Kathmandu University, Kathmandu, Nepal

${ }^{2}$ Lecturer, Department of Anatomy, IOM Ayurved Campus, Kritipur, Kathmandu, Nepal

${ }^{4}$ Lecturer, Department of Pharmacology, Kantipur Dental college Teaching Hospital \& Research

Center,Kathmandu University, Kathmandu, Nepal

${ }^{5}$ Assistant Professor, Department of Pathology, Kantipur Dental college Teaching Hospital \& Research

Center, Kathmandu University, Kathmandu, Nepal

\begin{abstract}
Kidneys develop in early in 5th week and start to function around 9th week in the intermediate mesoderm of human embryos as metanephric kidney. To correlate the chronological pattern of kidney development in foetuses of different gestational period in the geographical eastern region of India, Odisha \& compare the results from other researchers nationwide \& worldwide. Aborted human foetuses without obvious congenital anomaly of gestational age between 12 weeks and 36 weeks were collected and processed for histological sections by Haematoxylin and Eosin stain. In our present study we found that Kidney was covered by a thick capsule made up of fibrous tissue beneath which cortico-medullary differentiation was well marked. Glomerulus with afferent \& efferent arteioles were present in kidney. Proximal Convoluted Tubules, Distal Convoluted tubules, Collecting tubules \& Duct of Bellini visible in medulla. Cortico-medullary junction well differentiated \&Cortex is more than Medulla. Lobar \& interlobar arteries seen in renal corpuscles. Lobulation \& medullary rays visible in cortex. Between medullary rays multiple crescenteric glomeruli were visible. Bowman's Capsule \& subcapsular space present. These structures were absent in younger foetuses \& develop in increasing gestational age but the kidney in foetuses were lobulated which was absent successively. From the current study one can conclude that major part of development of fetal kidney occurs during midgestational period \& continues until the last week of 3 rd trimester of gestation.

Keywords: Metanephric Kidney, Proximal Convoluted Tubules, Distal Convoluted Tublules, Collecting Tubules.
\end{abstract}

\section{INTRODUCTION}

The emphasis on genetic counseling and possibility of early prenatal diagnosis has stimulated interest in fetal kidney anatomy ${ }^{1}$. During Embryogenesis Kidneys develop from the nephrogenic cord of the intermediate mesoderm of the human embryo. The development of Kidney starts as early in $5^{\text {th }}$ week of fertilization and is characterized by the successive development of prenephros, mesonephros and metanephros ${ }^{1}$. The prenephos and mesonephros are temporary while the metanephros exists and develop fully to 
become the prominent organ of the excretory system $^{3}$. Metanephros develops from three sources-metanephric diverticulum (ureteric bud) forming the collecting part $\&$ metanephric mass of intermediate mesoderm (metanephrogenicblastema) forms the secretory part ${ }^{1}$ and the angiogenic mesoderm giving rise to glomeruli and vasa recta. The study of the developmental pattern of the kidneys during foetal life will help to screen any congenital disorders present in the excretory system $^{4}$. The present study aims at studying the developmental changes that occur during the foetal life from 12 weeks to 36 weeks of gestation.

\section{MATERIALS \& METHODS}

The present study was carried out in the department of Anatomy, Hi-Tech Medical College \& Hospital, Bhubaneswar. The material for the study consisted of 32 aborted human fetal specimens from 12th to 36th weeks of gestational ages. The specimens were provided by the department of Obstetrics \& Gynaecology different Medical College \& Hospital, Bhubaneswar for routine fetal autopsy From May 2012 to February 2013. All fetuses were result of the intra uterine death or spontaneous abortion. Consent for autopsy and brief antenatal, medical, past history from the mother was taken from the parents to perform the study. The fetuses were divided into four groups according to the gestational age:

\section{Study Population}

A total number of 32 aborted human foetuses without obvious congenital anomaly of gestational age between 12 weeks and 36 weeks collected within 6 hours of delivery by spontaneous miscarriages \& therapeutic legal abortions. Study samples were arbitrarily divided into groups of biweekly gestational age by duration of amenorrhoea from medical records \& ultrasound photometry after receipt of informed consent from mother and legal guardians. The measurements of the glomeruli were taken according to Bridgette $\mathrm{J}$ et $\mathrm{al}^{5}$.

\section{Tissue Processing and Section Cutting:}

Foetuses were immediately fixed in $10 \%$ Formalin for 1-2 hrs. Kidneys were dissected by Dissecting Microscope, fixed in 10\% Formalin for 48-72 hrs. After fixation by formalin, the tissues were transferred to $30 \%, 50 \%, 70 \%, 90 \%$ and Absolute alcohol each for 30 minutes. This ascending grading of the dehydrating fluid was done because when alcohol mixes with water, it produces diffusing current which can damage the tissues. Then the tissues were put in xylol for 24 hours to clear the residual alcohol. These tissues were processed for paraffin sections by tissue blocking (Paraffin Embedding). 3 pots of hard paraffin were taken; paraffin was melted in the incubator at 56 degrees, as hard paraffin is ideal for materials which are to be cut in thin sections about 12 micron. The tissue was put in the first pot containing equal parts of paraffin and xylene and then changed to second and third pots containing only fresh melted paraffin at 90 minutes interval. Then the tissues were mounted in fresh melted paraffin with L-Block. The L-Block was then trimmed to a rectangular shape. Then the L-Block was fixed with the block holder (choke) and the block holder was clamped in the rotary microtome. $5 \mathrm{mu}$ sections were cut in rotary microtome. The microtome was revolved at 40 per min and ribbon was formed. Then the ribbon was put in tissue flotation bath. Albuminised slide was then made by putting a drop of Mayor's albumin (equal parts of glycerine and egg white) and spreading it uniformly by rubbing with finger. The piece of ribbon was then taken on the slide and dried at room temperature. The slide was then put in the warming table. When the paraffin melted the slide was put into xylene for 2-3 minutes because xylene removes paraffin.

\section{Staining:}

The tissue was put in decreasing grades of alcohol (Absolute alcohol,90\%,70\%,50\% and 30\%) then was put in the prepared Harris Alum Haematoxylin (nuclear) stain for 7 minutes and lastly washed with distilled water.2-3 drops of $1 \%$ acid alcohol (1cc $\mathrm{HCl}$ in $75 \%$ alcohol) was added 
to remove the excess stain beyond the nucleus. The slide was then put in running tap water for 30 minutes to develop haematoxylin colour (bluish). Then the slides were again dipped in ascending grades of alcohol $(30 \%, 50 \%$, and $70 \%)$ and then put in eosin Y (cytoplasmic) stain for 30 seconds. Then the slide was washed with absolute alcohol for a few seconds so that excess of eosin was removed and lastly the slide was placed in xylol. The slide was then taken out from xylene and then put in 1-2 drops of DPX (Adhesive agent) and a cover slip was put on it and pressed slightly so that air bubbles were removed. Sections were then seen in light microscope under low power $10 \mathrm{X}$ followed by high power $45 \mathrm{X}$ magnification. Thereafter photomicrographs were taken by camera using microscope adapter.

\section{RESULTS}

\section{Morphological observation}

All the kidneys taken for the study were found in lumber region- bilaterally and were lobulated in appearance.

\section{Microscopic findings}

12 weeks: Cortex \& Medulla couldn't be differentiated in the peripheral region. There were number of nephric vesicles formed by condensation of disorganised mesenchymal cells.

14 weeks: Lobes separated only in superficial part of cortex \& in deeper part of cortex they were fused with each other. Kidney covered by a thin capsule made up of fibrous tissue beneath which cortico-medullary differentiation was not well marked. Cortex is more than Medulla.

16 weeks: capsule of the Kidney was visible, just deep to it multiple glomerulus present in cortical part. Capsular space well visible.Bowman's Capsule\&subcapsular space present. Definite medulla present.

18 weeks: Medullary Rays visible Between medullary rays multiple cresenteric glomeruli visible. Medulla with Tubules visible. In 18 Weeks Fetal Kidney Lobules \& Capsule present. Medullary Rays visible in cortex. Both PCT \&
DCT clearly visible.Large blood vessels seen in medulla. Connective tissue in between tubules areplenty. Tubules arranged in groups seperatedby connective tissue in cortex. Peripheral Part of cortex renal corpuscles are smaller size but JuxtaGlomerular part of cortex renal corpuscles are larger in size.

20 Weeks: Renal cospuscles visible.Many blood vessels seen in medulla. Collecting tubules visible in medullary region. Medullary rays visible in cortex.

22 Weeks: Lobulation still present. Many blood vessels in medulla. Renal cospuscles more compact towards periphery of cortex.PCT \& DCT visible.

24 Weeks: Collecting tubules present in medullary region. Lobulation\& medullary rays were visible. Renal corpuscles in cortex. Thin capsule present

26 Weeks: Thin capsule visible below which developing glomerulus not well differentiated. PCT \& DCT present in deeper part of cortex with fully developed renal corpuscles. Lobulation are still present. Loops of Henle \& connective tissue visible. Occasionally some blood vessels are seen in the field.

28 Weeks: Nephrogenic zone thin under capsule. Large amount of blood vessels seen.Collecting tubules \& Loops of Henle seen in medullary region.

30 Weeks: Nephrogenic zone very reduced. Duct of Bellini\& collecting tubules clearly seen in medulla. PCT, DCT\& Loop of Henle seen. Cortico-medullary junction well differentiated. Vascular pole of renal cospuscles formation visible.

32 Weeks: Vascular pole exists in renal cospuscles. Capsule well demarcated. Nephrogenic zone is very thin. PCT, DCT, Collecting tubules \& Duct of Bellini visible.

34 Weeks: Glomerulus with afferent\& efferent arterioles visibles. Other findings are similar.

36 Weeks: Cortex is more than Medulla. Lobar \& interlobar-arteries seen. Other findings are similar. 

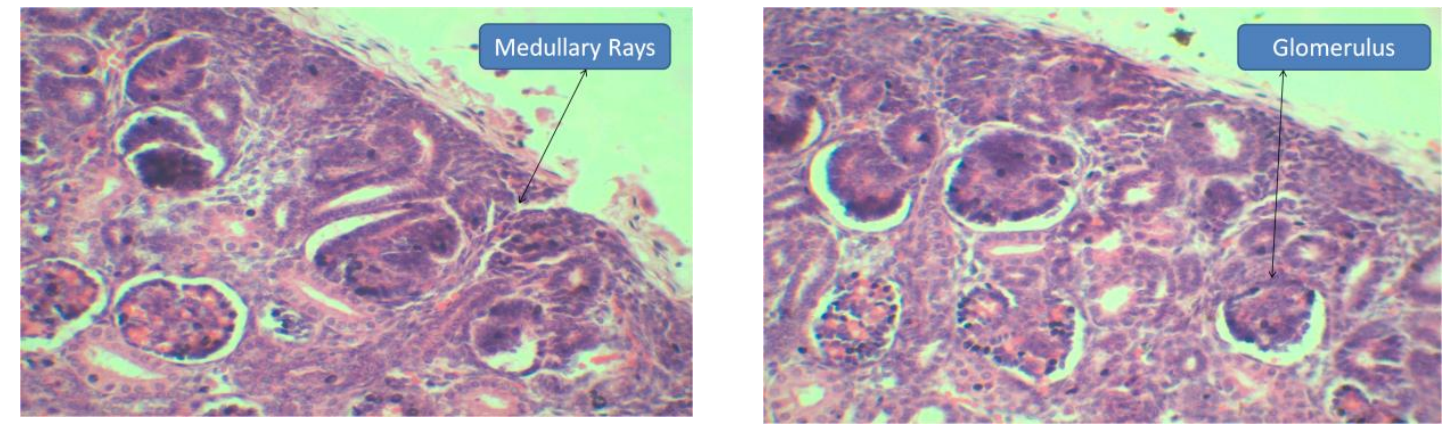

Table No. 1- OBSERVATIONS (Left Kidney)

\begin{tabular}{|c|c|c|c|c|}
\hline WEEKS & LENGTH (in cm) & BREADTH (in cm) & WIDTH (in cm) & WEIGHT (in $\mathrm{gm}$ ) \\
\hline 10 & 1.1 & 0.5 & 0.3 & 0.2 \\
\hline 12 & 1.2 & 0.6 & 0.6 & 0.31 \\
\hline 14 & 1.4 & 0.9 & 0.7 & 0.6 \\
\hline 16 & 1.4 & 0.8 & 0.6 & 0.9 \\
\hline 18 & 1.8 & 0.9 & 0.8 & 1.0 \\
\hline 20 & 1.3 & 0.9 & 0.7 & 1.08 \\
\hline 22 & 1.6 & 0.8 & 0.9 & 1.2 \\
\hline 24 & 0.9 & 1.0 & 1.0 & 1.8 \\
\hline 26 & 2.0 & 1.2 & 1.0 & 0.6 \\
\hline 28 & 1.9 & 1.0 & 0.9 & 1.5 \\
\hline 30 & 2.0 & 1.5 & 0.8 & 1.9 \\
\hline 32 & 3.4 & 2.1 & 1.6 & 2.9 \\
\hline 34 & 3.9 & 2.7 & 1.8 & 3.8 \\
\hline 36 & 4.7 & 3.2 & 2.1 & 4.3 \\
\hline 38 & 5.2 & 3.6 & 2.8 & 4.9 \\
\hline
\end{tabular}

Graph 1: OBSERVATIONS (Left Kidney)

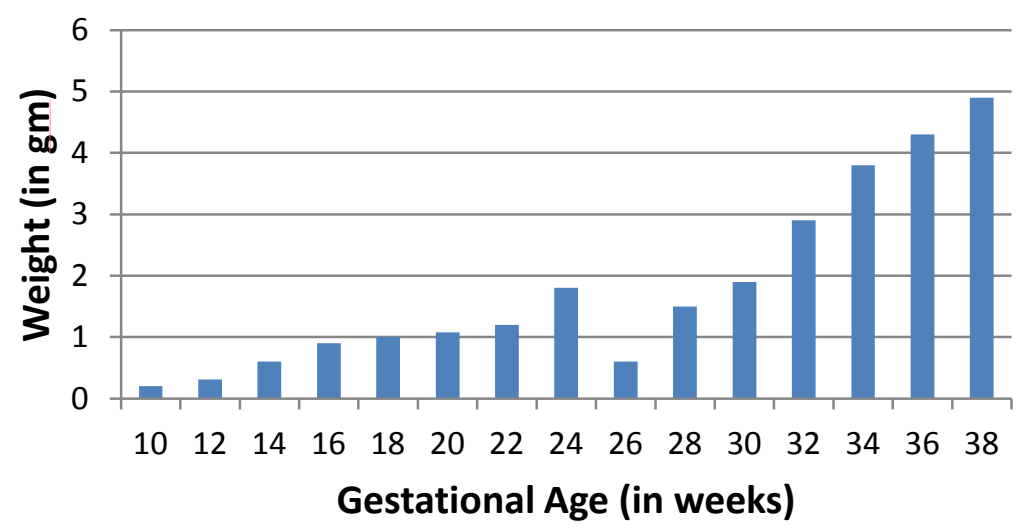

Table 2 OBSERVATIONS (Right Kidney)

\begin{tabular}{|c|c|c|c|c|}
\hline WEEKS & LENGTH (in cm) & BREADTH (in cm) & WIDTH (in cm) & WEIGHT (in $\mathrm{gm}$ ) \\
\hline 10 & 1.0 & 0.6 & 0.4 & 0.22 \\
\hline 12 & 1.0 & 0.6 & 0.6 & 0.3 \\
\hline 14 & 1.4 & 1.0 & 0.9 & 0.7 \\
\hline 16 & 1.2 & 0.7 & 0.6 & 0.88 \\
\hline 18 & 1.4 & 0.7 & 0.8 & 1.18 \\
\hline 20 & 1.4 & 0.8 & 0.8 & 1.2 \\
\hline 22 & 1.5 & 0.9 & 0.7 & 1.17 \\
\hline 24 & 1.0 & 0.8 & 0.6 & 1.7 \\
\hline 26 & 1.7 & 1.3 & 1.1 & 0.6 \\
\hline 28 & 1.0 & 1.1 & 0.9 & 1.45 \\
\hline 30 & 2.0 & 1.6 & 0.8 & 2.0 \\
\hline 32 & 3.2 & 1.9 & 1.7 & 2.7 \\
\hline 34 & 3.7 & 2.2 & 1.9 & 3.2 \\
\hline 36 & 4.3 & 2.8 & 2.3 & 3.9 \\
\hline 38 & 5.1 & 3.2 & 2.5 & 4.2 \\
\hline
\end{tabular}


Graph 2 OBSERVATIONS (Right Kidney)

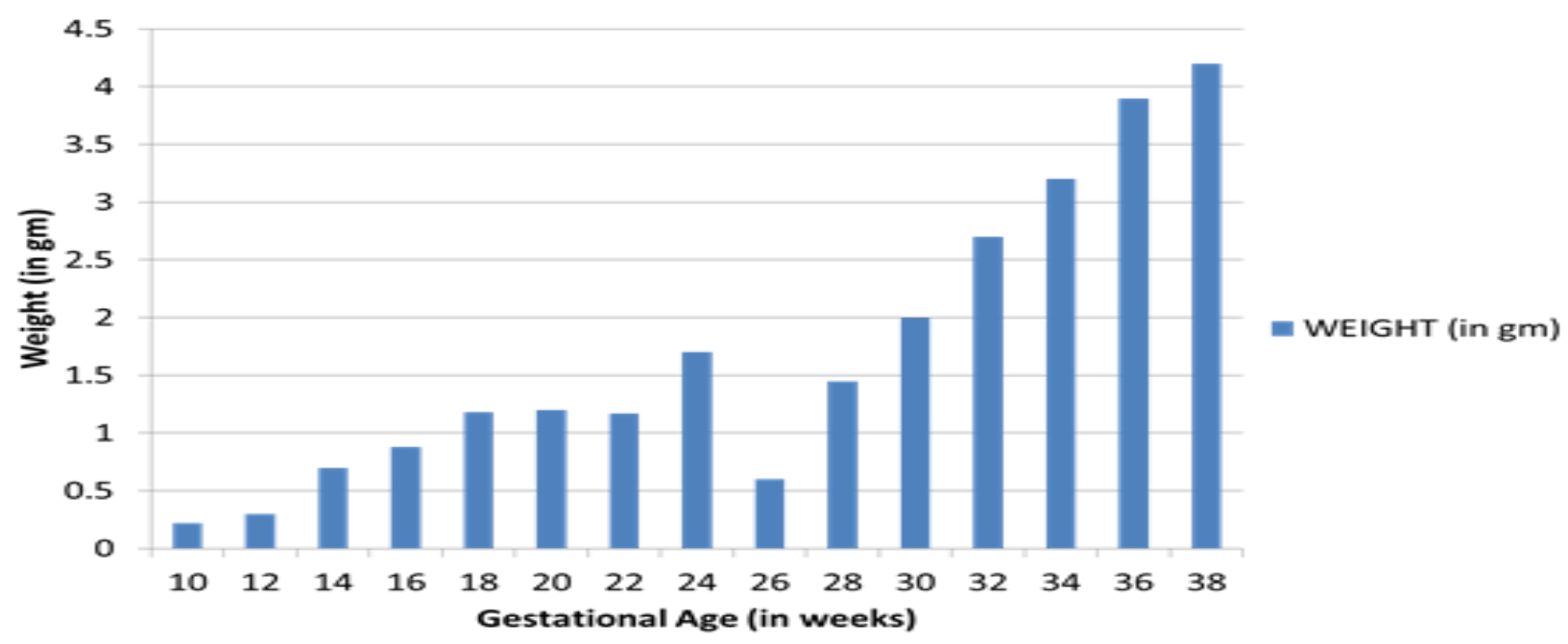

\section{DICUSSION}

The human foetal kidneys under microscope showed well marked lobulation in the early weeks of fertilization, but as the weeks of fertilization increased the lobulations less marked. Similarly, the lobes were found to be separated only in the superficial part of cortex while in the deep they remained fused. Kidney was covered by a thin capsule made up of fibrous tissue. During the early weeks of gestation only immature glomeruli was present beneath the capsule. In our study we found that the cortico-medullary differentiation was not well marked in the early weeks of gestation but in the later weeks the differentiation could be well observed under the microscope. Similar findings were reported by the study of Daković-Bjelaković M et al ${ }^{6}$.

The thickness of cortex and medulla increased gradually with increase in gestation period, however, the nephrogenic zone's size decreased. Deep to nephrogenic zone, but in the superficial part of cortex, the various stages of developing glomeruli were seen in different stages of development. In parallel with the study of Emery J.L ${ }^{7}$ scattered glomeruli were seen near the arcuate vessels.Study suggests that scattered glomeruli in the arcuate vessels represent involution forms of large glomeruli which formed early in this region in intra-uterine life and possibly represent a transitory renal structure ${ }^{7}$.
In our present study we found that size of glomeruli increased as we moved from outer zone of cortex to middle and inner zone.Our present findings show that the size of the glomeruli in respective regions is quite similar in various age groups and it is in accordance with the findings of Shimada $\mathrm{K}$, et $\mathrm{al}^{8}$. At later stages of gestation the mature glomeruli appeared and their number increased in deeper parts of cortex. Similarly, we found that at the stage of 17 weeks of gestation the earlier developing tubules within the connective tissue fully differentiated into proximal and distal convoluted tubules. Furthermore, the undifferentiated mesenchymal tissue content of the Medulla decreased, number of mature tubules increased while the connective tissue mass decreased as the kidney matured. We also observed prominent vascularisation of both cortex and medulla at the later stages of gestation.

\section{CONCLUSION}

These finding may prove useful in defining fetal kidney diseases such as agenesis, hypoplasia, multicytic kidney, polycystic kidney etc. more precisely using the most modern invasive or noninvasive imaging technique. From the current study one can conclude that major part of development of fetal kidney occurs during midgestational period \& continues until the last week of 3rd trimester of gestation. 


\section{BIBLIOGHAPHY}

1. Sampaio, F.J. Analysis of kidney volume growth during the fetal period in human. J. Urol. 2007;178(4Pt2):1570-4.

2. Tank KC, Saiyad SS, Pandya AM , Akbari VJ , Dangar KP A study of histogenesis of human fetal kidney. Int $\mathbf{J}$ Biol Med Res. 2012; 3(1): 1315-1321

3. KIRSI SAINIO* and ANNE RAATIKAINEN-AHOKAS. Mesonephric kidney - a stem cell factory? Int. J. Dev. Biol. 43: 435-439 (1999)

4. HIndRyCKx A, CATTe L. Prenatal diagnosis of congenital renal and urinary tract malformations. ObGyn, 2011, 3 (3): 165-174

5. Bridgette J. Mc Namara et. al. Association between age, body size and nephron number with individual glomerular volumes in urban west African males. Nephrol dial Transplant (2009) ; 24: 15001506

6. Daković-Bjelaković MVlajković S, Cukuranović R, Antić S, Bjelaković G, Mitić D. Quantitative analysis of the nephron during human fetal kidney development. Vojnosanit Pregl. 2005; 62(4): 281-286.

7. Emery J. L., Macdonald M. S., Involuting and scattered glomeruli in the kidneys of infants. American journal of pathology. 1960; 36: 713-722

8. Shimada K,Hosokawa S., Tohda A. Nippon Hinyokika Gakkai Zasshi. 1993; 84(12): 2091-2096.

\section{CORRESPONDING AUTHOR}

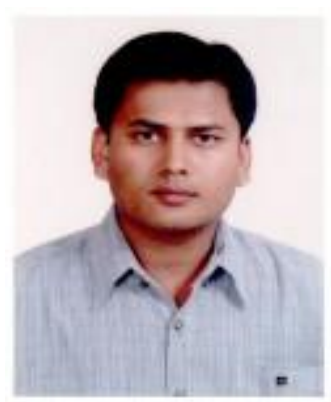

Mr. Ramendra Raman. Lecturer, Department of Anatomy, Kantipur Dental college Teaching General \& Dental Hospital \& Research Center, Kathmandu University, Kathmandu, Nepal. Email: ramanramendra@hotmail.com, Contact: +9779844174303 